OnLine Journal of Biological Sciences 10 (2): 66-72, 2010

ISSN 1608-4217

(C) 2010 Science Publications

\title{
Design and Evaluation of Three Pair Primers for Exon 1 Amplification of Hyaluroglucosaminidase-1 Gene
}

\author{
Usman Sumo Friend Tambunan, Sylvia Sugito and Arli Aditya Parikesit \\ Department of Chemistry, Faculty of Mathematics and Natural Science, \\ University of Indonesia, Depok 16424, Indonesia
}

\begin{abstract}
Problem statement: Hyaluronidase is an enzyme which catalyze hydrolysis of Hyaluronan (HA). Hyaluronan is important in cell migration during embryonic development, cellular proliferation and differentiation and has a structural role in connective tissues. Hyaluronidase deficiency is correlated with mucopolysaccharidosis IX. In human, hyaluronidase is encoded by HYAL1 gene. The mutation study of HYAL1 gene was carried out by many researchers, but until now, mutation study of HYAL1 still in progress and limited due to the lack of primer used in amplification of selected DNA sequence of HYAL1 gene and maximum length limitation imposed by DNA sequencer. Approach: The search for three pairs of primers for human exon 1 segmented amplification of HYAL1 gene was conducted and evaluated. The first step was to acquire HYAL1 gene sequence and then had it aligned with human chromosome 3 genomic contig sequence. Exon 1 of HYAL1 gene was found to be located at nt 201-1711 of the acquired human chromosome 3 genomic contig. Online Primer3 program was used to design three pairs of primers. The selected pairs of primer had been subjected to BLASTn operation for selectivity examination while onlineNetPrimer operation was carried out for examination of secondary structures. Results: The search for primers to amplify three different fragments of exon 1 of HYAL1 gene yielded three selected pairs of primers, namely forward primer 5'-TGACCCCCTACAAAAGCTCA-3' (20 bp) and reverse primer 5'AAGTCTCCGATTCCCCCACT-3' (20 bp) for amplifying nt 1-551 of HYAL1 gene, forward primer 5'-AGTCCTGTGGGAGATGGCAGA-3' $(21 \mathrm{bp})$ and reverse primer 5'CGGTAAATGTCCTTGGTGTCC-3' (21 bp) for amplifying nt 355-1053 of HYAL1 gene and forward primer 5'-GCCATACCTGCTCCTGACTT-3' $(20 \mathrm{bp})$ and reverse primer 5'ACAAGGTGGGCAGGTTACAG-3' (20 bp) for amplifying nt 956-1511 of HYAL1 gene. When using these primers, nt 1-46 of amplified product of the first pair of primers and nt 555-584 of amplified product of the third pair of primers must not be considered because those are not part of exon 1 of HYAL1 gene. The results from both operations and trial to real samples using these primers indicated that all three pairs of primer were satisfactory for use. Conclusion/Recommendations: The three pairs of primers could be used to amplify specified segments of HYAL1 gene.
\end{abstract}

Key words: HYAL1 gene, exon 1, human chromosome 3, primer design, PCR

\section{INTRODUCTION}

Hyaluronan (HA) is a negatively charged glycosaminoglycan with high molecular weight and an important extracellular matrix component. It consist repeating units of disaccharide and does not contain any sulfate functional group (Stern, 2004). It is found in connective tissues, epidermis, cartilage, synovial fluid and is available throughout the rapid regeneration of tissue (Frost et al., 1997). HA serves as a joint lubricant and is also implicated in cell proliferation, differentiation, cell-cell recognition, tumor growth and invation (Chao et al., 2007; Simpson and Lokeshwar, 2007; Wang et al., 2008), angiogenesis (Chao et al., 2007; Liu et al., 1996) and inflammatory responses (Chao et al., 2007). Human HYAL1 is a lysosomal enzyme responsible for the hydrolysis of intracellular $\mathrm{HA}$ and is also detected in the plasma and urine (Csoka et al., 1998). Increased expression of HYAL1 mRNA and higher levels of HYAL1 are linked to the human genetic disorder mucopolysaccharidosis IX (Barbara et al., 1999).

Hyaluronan is locally catabolized. Internal degradation occurs after endocytosis and then

Corresponding Author: Usman Sumo Friend Tambunan, Department of Chemistry,

Faculty of Mathematics and Natural Science, University of Indonesia, Depok 16424, Indonesia 
immediately followed up with catalytic process by hyaluronidase enzyme (EC3.2.1.35) (Barbara et al., 1999). Hyaluronidase may be divided into two categories, each consisting of three members. The first group is located in chromosome 3p21.3 consisting of HYAL1, HYAL2 and HYAL3 gene (Barbara et al., 1999), while the other is located in chromosome 7q31.3 consisting of HYAL4, SPAM1 and HYALP1 gene. In human genome, HYAL1 is divided into three exons and extends to a size of $3.7 \mathrm{~kb}$.

Among hyaluronidase encoding gene, HYAL1 is the first to be ever isolated and characterized (Frost et al., 1997; Laurent et al., 1995). This was done by purifying hyaluronidase from human plasma and having its amino acid sequence matched with a particular cDNA in Expressed Sequence Tag (EST) database. It was then cloned, sequenced and expressed. This $57 \mathrm{kDa}$ acid-active enzyme comprises a single polypeptide chain of $49 \mathrm{kDa}$ with an additional $8 \mathrm{kDa}$ added by post-translational glycosylation. It is approximately $40 \%$ identical to the sperm-specific hyaluronidase PH-20 (Frost et al., 1997).

Mutations of HYAL1 related to mucopolysaccharidosis IX has been studied by using several sense and antisense primers used for amplification of fragments of HYAL1 (Barbara et al., 1999).

Amplification of human exon 1 of HYAL1 gene was using only one pair of primers, it is impossible for having more, due to DNA sequencer length limitation. Some studies (Afify et al., 1993) show that designing another of primers was important in order to cover the complete sequence of exon 1 of HYAL1 gene. The aim of this study was to search and design primers that can be used to amplify fragment exon 1 of HYAL1 gene in humans as an additional information to complete the result from previous-studies (Barbara et al., 1999).

\section{MATERIALS AND METHODS}

Design and evaluation of primers: Complete human HYAL1 gene sequence was acquired from the website of National Centre for Biotechnology Information (NCBI) (http://www.ncbi.nlm.nih.gov.). The search for HYAL1 gene DNA sequence of human chromosome 3 genome contig was also conducted. The exact location of HYAL1 gene in human was determined by aligning HYAL1 gene with human chromosome 3 genome contig. Analysis was immediately directed on exon 1 of HYAL1 gene. Primer candidates in three partially overlapping sequences of exon 1 of HYAL1 gene were thoroughly searched using online primer3 accessed at http://frodo.wi.mit.edu/cgibin/primer3/primer3_www.cgi. BLASTn operation was conducted to the best candidate pair of primers in each of the partially overlapping sequences of exon 1 of HYAL1 gene by accessing http://www.ncbi.nlm.nih.gov/BLAST/blast.cgi. This operation was to examine the candidates pair of primers for homological similarity with other genes in the GenBank.

In addition to BLASTn operation, the primers were examined for secondary structures by using online NetPrimer provided by Premier Biosoft. This operation examined the primers for secondary structures such as hairpin, dimer, cross dimer, palindrome, repeat and runs. The selected pairs of primers were that which had the least secondary structures, or none at all.

Blood sampling: Blood samples from 3 healthy individuals at the age range of 20-30 yearswere collected at Central Operation Installation and Pediatric Ophthalmology division of RSUPNCM, Faculty of Medicine University of Indonesia. Healthy individuals were screened as not suffering from any disease during blood sampling. Blood sampling were executed by carrying out intravenous injection using a terumo syringe with a $23 \mathrm{G}$ needle attached to a vaccutainer containing 2 cc volume of EDTA as anticoagulant. The vaccutainers containing the blood samples were then placed into an icebox. The blood samples were either used as prepared or stored in refrigerator for no later than 7 days prior to usage (Lachaud et al., 2000).

Preparation of DNA solutions: DNA isolation from these blood samples were performed with Wizard®Genomic DNA Purification Kit consisted of cell lysis solution, nucleic lysis solution, protein precipitation, DNA rehydration and RNAse $\left(10 \mathrm{mg} \mathrm{mL}^{-1}\right)$. The steps of DNA isolation were carried out according to manufacturer's instruction (Kim et al., 2009).

Dilution of primers: Each and every primer was diluted to a volume of $100 \mu \mathrm{L}$, where $1 \mu \mathrm{L}$ of the primer solution will represent a primer amount of 20 pmol. For the first pair of primer, $11 \mu \mathrm{L}$ of forward primer 5'GACCCCCTACAAAAGCTCA-3' (20 bp) was diluted with $89 \mu \mathrm{L} \mathrm{ddH}_{2} \mathrm{O}$ and $12.49 \mu \mathrm{L}$ of reverse primer 5'AAGTCTCCGATTCCCCCACT-3' (20 bp) diluted with $89.51 \mu \mathrm{L} d \mathrm{ddH}_{2} \mathrm{O}$. For the second pair of primer, $9.1 \mu \mathrm{L}$ of forward primer 5'-AGT CCTGTGGGAGATGGCAGA-3' (21 bp) diluted with $90.9 \mu \mathrm{L} \mathrm{ddH}_{2} \mathrm{O}$ and $9.74 \mu \mathrm{L}$ reverse primer 5'CGGTAAATGTCCTTGGTGTCC-3' (21 bp) was diluted with $90.26 \mu \mathrm{L} \mathrm{ddH}_{2} \mathrm{O}$. For the third pair of primer, 6.6 $\mu \mathrm{L}$ of forward primer 5'GCCATACCTGCTCCTGACTT-3' (20 bp) diluted 


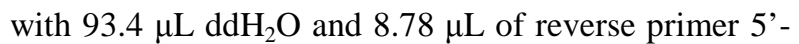
ACAAGGTGGGCAGGTTACAG-3' (20 bp) diluted with $91.22 \mu \mathrm{L} \mathrm{ddH}_{2} \mathrm{O}$.

PCR amplification: The amplification process utilized the Thermocycler Perkin Elmer GeneAmp® PCR System 9700 and components from PromegaPCR Core systems. Mixture amounting to $50 \mu \mathrm{L}$ in an eppendof tube of $0.2 \mathrm{~mL}$ consisted of $\mathrm{MgCl}_{2} 1.5 \mathrm{mM}(3 \mu \mathrm{L})$, $\mathrm{MgCl}_{2}$-free buffer solution of thermophilic DNA polimerase reaction (for 10 reactions) $(5 \mu \mathrm{L})$, PCR Nucleotide Mix $200 \mu \mathrm{M}(1 \mu \mathrm{L})$ and 20 pmol of forward and reverse primer $(@ 1 \mu \mathrm{L})$.

Later, Tag DNA polymerase $1.25 \mathrm{U}(0.25 \mu \mathrm{L})$, $1650-15300 \mathrm{ng}(15 \mu \mathrm{L})$ of DNA template and sterile of $\mathrm{ddH}_{2} \mathrm{O}(23.75 \mu \mathrm{L})$ were then added to the mixture to a final volume of $50 \mu \mathrm{L}$. The whole process was carried out in an ice bath. The cold mixture was then placed in PCR equipment that had been preconditioned for 15 min. Then, exon 1 fragment amplification of HYAL1 was allowed to proceed. At the initial step of the process, strains of DNA were allowed to denaturize at $95^{\circ} \mathrm{C}$ for $5 \mathrm{~min}$, after which the first cycle of every step denaturation was carried out for only $1 \mathrm{~min}$ at the same temperature. Throughout the process, DNA annealing was carried out at lowered temperature of $62^{\circ} \mathrm{C}$ for $1 \mathrm{~min}$ and was immediately followed by DNA extension for $1 \mathrm{~min}$ at $72^{\circ} \mathrm{C}$. The whole process was carried out for 35 cycles. In end of the process, at the 35 th cycle, DNA extension was carried out for $7 \mathrm{~min}$, after which the temperature of mixture was then dropped to $4^{\circ} \mathrm{C}$.

Electrophoresis on PCR products: Electrophoresis was carried out using Mini DNA Electrophoresis integrated with Biorad Power Supply (model 200/2.0). Agarose gel 2\% was used. This was prepared by adding to $1 \mathrm{~g}$ of agarose powder, $50 \mathrm{~mL}$ of TAE $1 \mathrm{X}$ prepared by adding $980 \mathrm{~mL}$ of $\mathrm{ddH}_{2} \mathrm{O}$ to $20 \mathrm{~mL}$ of TAE $50 \mathrm{X}$. The mixture was heated in a microwave and was later cooled to a temperature of $60^{\circ} \mathrm{C}$ and added $0.5 \mu \mathrm{L}$ of etidium bromide. The liquid mixture was then poured into a cast, which contained a comb-like structure. The liquid mixture in the cast was allowed for cooling down to room temperature. After the agarose gel of $2 \%$ was ready for use, $5 \mu \mathrm{L}$ of PCR product mixed with tracking dye $(0.25 \%$ blue bromophenol, cyanolxylene, sucrose $(4 \% \mathrm{~b} / \mathrm{v}))$ was loaded to the electrophoresis apparatus well. Separation of PCR product was carried out by subjecting the apparatus to a 90 volt of voltage for 45 min with the cathode was positioned near the well. The DNA molecular weight marker was 100 bp DNA ladder. The electrophoresis product was visualized with a long life ${ }^{\mathrm{TM}}$ Spectroline ${ }^{\circledR}$ UV transluminator and was promptly photographed using a Polaroid camera (Shuttleworth et al., 2002).

\section{RESULTS}

By accessing http://www.ncbi.nlm.nih.gov, complete sequence of Homo sapiens Hyaluroglucosaminidase-1 (HYAL1) gene, 3679 bp in size, was acquired by the Gene Identifier code of 2314819 (Fig. 1).

By following the same process, incomplete sequence of chromosome 3 genome contig, 3912 bp in size, which was the estimated locus of HYAL1 gene in human, was acquired by the Gene Identifier code of 51464027 (Fig. 1).

In order to find the exact location of HYAL1 gene in human chromosome 3-genomecontig, BLAST of nucleotide (BLASTn) operation was conducted to both HYAL1 gene and chromosome 3-genome contig. The result determined that HYAL1 gene is embedded into 3 exons in human chromosome 3-genome contig. With respect to this incomplete chromosome 3 genome contig acquired at the website of NCBI, the first exon is embedded in nt 1-107 (exon 1a) and 593-1516 (exon 1b), the second exon is in nt 2496-2585, while the third is in nt 2773-3679 (Fig. 2). This study will focus on exon 1 of HYAL1 gene.

In order to search three pairs of primers for amplifying whole sequence of exon 1 of HYAL1 gene in humans, three partially overlapping sequence of chromosome 3 genome contig were selected, namely nt 121-840, 541-1380 and 781-1920 (Fig. 3) of chromosome 3 genomic contig. These sequences were made overlapping in order to cover all of HYAL1 gene and to anticipate mutations in annealing region of one of the three pairs of primers, i.e., the HYAL1 gene sequence was not apriori considered to be conserved.

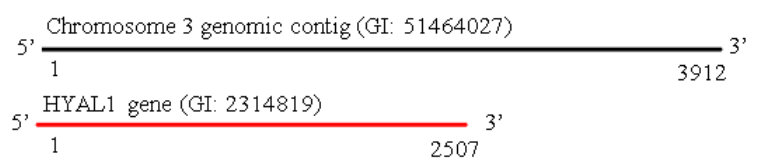

Fig. 1: HYAL1 gene and human chromosome genomic contig

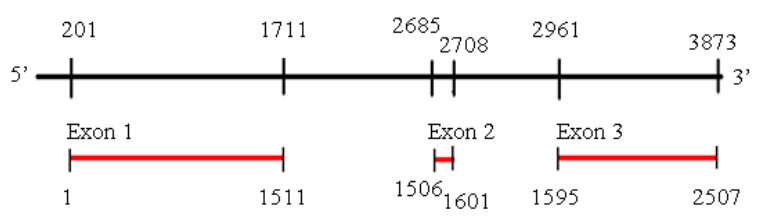

Fig. 2: Location of HYAL1 gene in human chromosome 3 genomic contig 


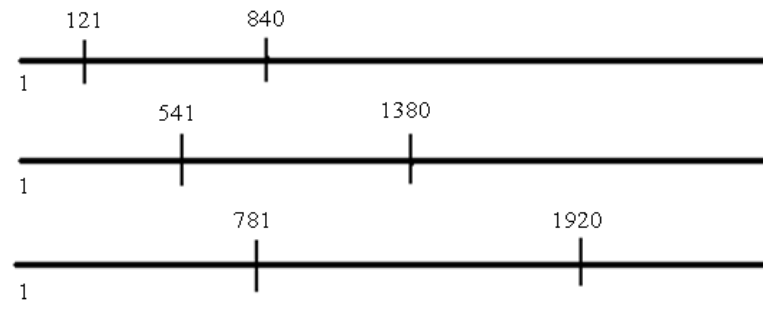

Fig. 3: Three studied human chromosome 3 genomic contig sequences in the search for three pairs of primers

By using primer3 software accessed from http://www.genome.wi.mit.edu/cgi-bin/primer/primer3 www.cgi, it was found that there were 1995 options of forward primers and 1924 options of reverse primers in nt 121-840. From software evaluation, only 60 forward primers met specifications as good quality primers. The other primers were excluded due to bad GC\%, low melting temperature and due to other criteria. From the 1924 reverse primers, only 60 met specifications. Pairing statistics shows that there was 2327 pairing considered, but only 7 were determined to be worth pairing. For nt 541-1380, there were 2190 options of forward primers and 2763 options of reverse primers. Only 31 forward primers and 69 reverse primers met specifications as good quality primers. Pairing statistics shows that there was 3297 pairing considered, but only 6 were determined to be worth pairing. For nt 7811920, there were 3627 options of forward primers and 1833 options of reverse primers. Only 330 forward primers and 311 reverse primers met specifications as good quality primers. Pairing statistics shows that there were 129 pairing considered, but only 8 were determined to be worth pairing.

Based on the above evaluation, three best pairs candidates of primers were selected. In the case of region nt 121-840, forward primer 5'TGACCCCCTACAAAAGCTCA-3 ${ }^{\prime} \quad(20$ bp $)$ and reverse primer 5'-AAGTCTCCGATTCCCCCACT-3' $(20 \mathrm{bp})$ were selected; forward primer 5'AGTCCTGTGGGAGATGGCAGA-3' (21 bp) and reverse primer 5'-CGGTAAATGTCCTTGGTGTCC-3' (21 bp) were selected in the region nt 541 to 1380; and forward primer 5'-GCCATACCTGCTCCTGACTT-3' $(20$ bp) and reverse primer 5'ACAAGGTGGGCAGGTTACAG-3' (20 bp) were selected in the region nt 781-1920. Amplified fragments of exon 1 HYAL1 sequence by these three pairs of primers is expected to be representative of exon 1 HYAL1 gene.
After choosing the above mentioned three pairs of primers, the specificity of primers candidates were examined using BLAST program that was accessed at http://www.ncbi.nlm.nih.gov/BLAST/. The primer sequences were set as input to BLAST of nucleotide (BLASTn), which determined their homology to human genome. It wasthoroughly examined. The primers were expected to exhibit little homology to human genome, so that they will direct themselves to anneal to specific area of fragments that will be amplified. By carrying out this process, each selected pair of primer was ensured to be specific enough for amplification of just one particular sequence of exon 1 of HYAL1 gene sequence. The forward primer from the first pair of primers yielded 6 homologies with human genome, while the reverse primer yielded 4 homologies; the forward primer from the second pair yielded 40 homologies, while the reverse primer yielded 36 homologies; the forward primer from the last pair yielded 30 homologies, while the reverse primer yielded 34 homologies.

\section{DISCUSSION}

After the homology examination, secondary structures were evaluated using online Netprimer that was accessed at http://www.primerbiosoft.com/netprimer/netprlaunch/ netprlaunch.html. The preferred parameters were $250.0 \mathrm{pM}$ for nucleic acid concentration, $50.0 \mathrm{mM}$ for mono ion concentration, $1.5 \mathrm{mM}$ for $\mathrm{Mg}^{2+}$ concentration, $204.92 \mathrm{mM}$ for total $\mathrm{Na}^{+}$concentration and temperature set at $25.0^{\circ} \mathrm{C}$. The forward primer from the first pair has no hairpin structure, two dimers (Fig. 4), no cross dimer, no palindrome, two runs (CCCCC and AAAA) (Fig. 4), no repeats, while the reverse primer has no hairpin structure, 1 dimer (Fig. 5), no palindrome, 1 run (CCCCC) (Fig. 5) and no repeats. The forward primer of the second pair has 2 hairpin structures (Fig. 6), 1 dimer, no palindrome, 1 run (GGG) (Fig. 6) and no repeats, while the reverse primer has no hairpin, no dimer, no palindrome, 1 run (AAA) (Fig. 7) and no repeats. This second pair of primers has 1 cross dimer (Fig. 8). The forward primers of the last pair has no hairpin, no dimer, no palindrome, runs or repeat (Fig. 9), while the reverse primer has no hairpin, no dimer, has no palindrome, has 1 runs (GGG) (Fig. 10) and has no repeat. The last pair of primer has 6 cross dimers (Fig. 11). In the above stated parameters, the first, second and third forward primer will have a $\mathrm{T}_{\mathrm{m}}$ of $58.03,61.59$ and $56.67^{\circ} \mathrm{C}$ respectively, while the first, second and third primer have a $\mathrm{T}_{\mathrm{m}}$ of $58.8,59.35$ and $57.36^{\circ} \mathrm{C}$ respectively. 
OnLine J. Biol. Sci., 10 (2): 66-72, 2010

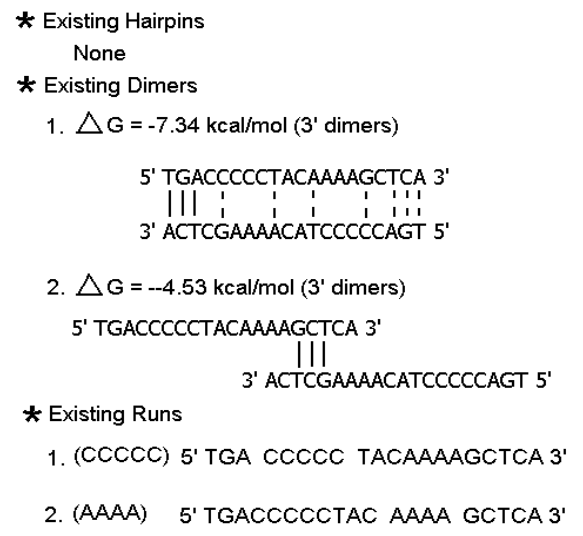

Fig. 4: Evaluation of forward primer for amplifying nt 1-551 sequence of exon 1 of HYAL1 gene

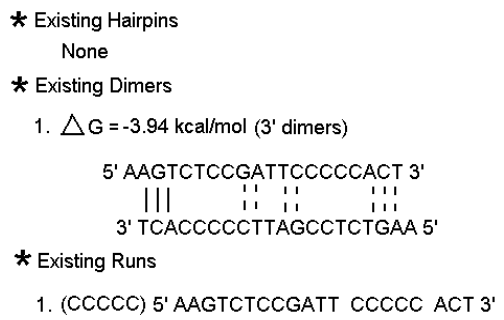

Fig. 5: Evaluation of reverse primer for amplifying nt 1-551 sequence of exon 1 of HYAL1 gene

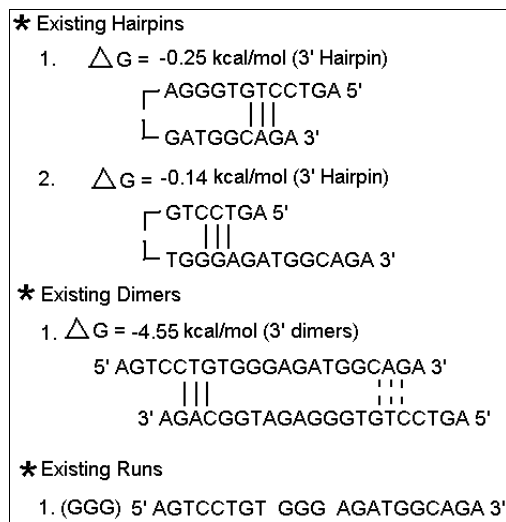

Fig. 6: Evaluation of forward primer for amplifying nt 355-1053 sequence of exon 1 of HYAL1 gene

The forward primer of the first pair was given a rating of 86 from a total score of 10 , while the reverse primer was given a rating of 92 ; the forward primer of the second pair was given a rating of 91 , while the reverse primer was given a rating of 100; both forward and reverse primer of the third pair was given a rating of 100 .

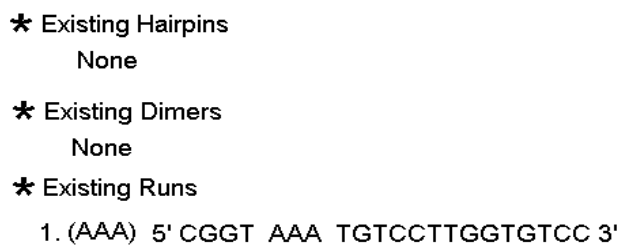

Fig. 7: Evaluation of reverse primer for amplifyingnt 355-1053 sequence of exon 1 of HYAL1 gene

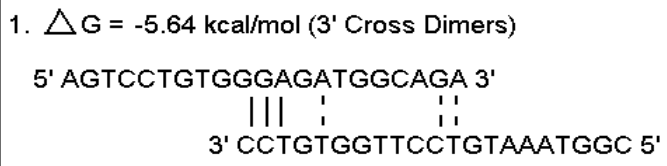

Fig. 8: Evaluation for existing cross dimer in the pair of primers for amplifyingnt 355-1053 sequence of exon 1 of HYAL1 gene

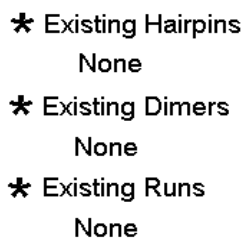

Fig. 9: Evaluation of forward primer for amplifying nt 956-1511 sequence of exon 1 of HYAL1 gene

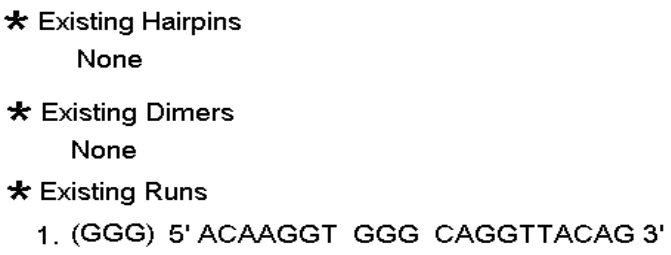

Fig. 10: Evaluation of reverse primer for amplifyingnt 956-1511 sequence of exon 1 of HYAL1 gene

The first pair of primer will amplify a product that is 597 bases in size. The amplified sequence is nt 155751 of human chromosome 3 genome contig. Sequence nt 155-200 is not part of HYAL1 gene (Fig. 12). So, in using these pair of primers, nt 1-46 of the amplified product must not be considered. The second pair will amplify a product that is 699 bases in size. The amplified sequence is nt 555-1253 of human chromosome 3 genome contig (Fig. 13). The last pair will amplify a product that is 584 bases in size. 
OnLine J. Biol. Sci., 10 (2): 66-72, 2010

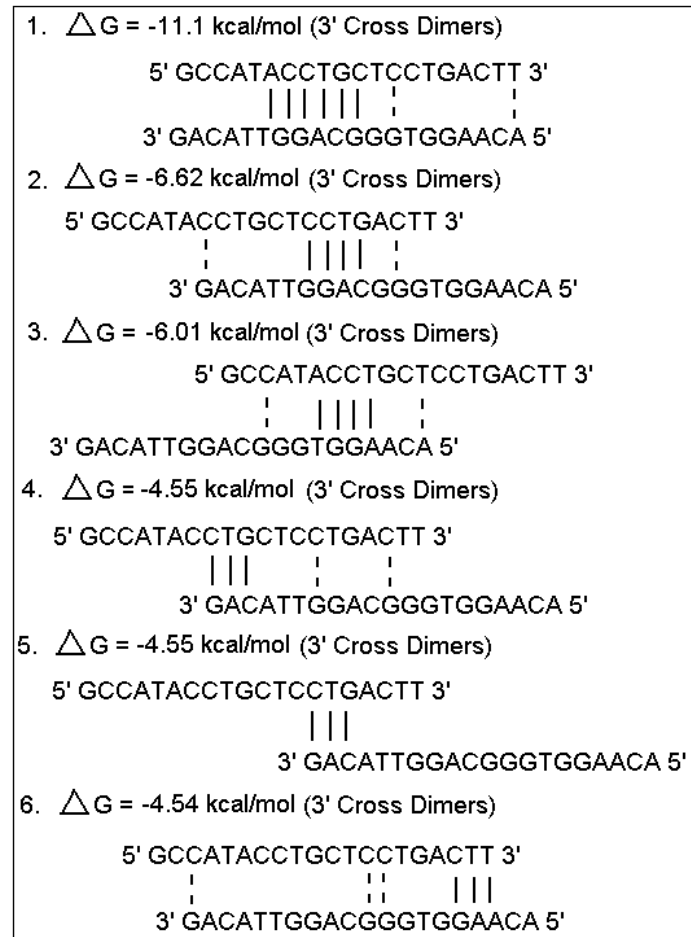

3. $\triangle \mathrm{G}=-6.01 \mathrm{kcal} / \mathrm{mol}$ (3' Cross Dimers) $5^{\prime}$ GCCATACCTGCTCCTGACTT $3^{\prime}$$$
\text { i }||||
$$

4. $\triangle \mathrm{G}=-4.55 \mathrm{kcal} / \mathrm{mol}$ (3' Cross Dimers)

5' GCCATACCTGCTCCTGACTT $3^{\prime}$

l | ! !

3' GACATTGGACGGGTGGAACA 5'

5. $\triangle \mathrm{G}=-4.55 \mathrm{kcal} / \mathrm{mol}$ (3' Cross Dimers)

5' GCCATACCTGCTCCTGACTT 3 '

I I I

3' GACATTGGACGGgTGGAACA 5'

6. $\triangle \mathrm{G}=-4.54 \mathrm{kcal} / \mathrm{mol}$ ( $3^{\prime}$ Cross Dimers)

$5^{\prime}$ GCCATACCTGCTCCTGACTT 3

$\begin{array}{llll}1 & 1 \\ 1 & 1\end{array}$

3' GACATTGGACGGGTGGAACA 5

Fig. 11: Evaluation for existing cross dimer in the pair of primers for amplifying nt 956-1511 sequence of Exon 1 of HYAL1 gene

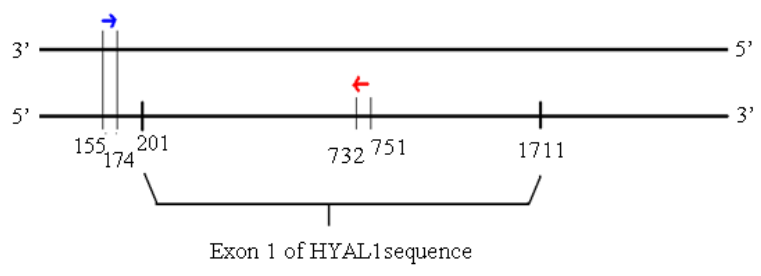

Fig. 12: Annealing region of the first pair of primers

The amplified sequence is nt 1156-1740 of human chromosome 3 genome contig. Sequence nt 1712-1740 is not part of HYAL1 gene (Fig. 14). So, in using these pair of primers, nt 555-584 of the amplified product must not be considered. With respect to HYAL1 gene, the first, second and third pair of primers will amplify nt 1-551, 355-1053 and 956-1511 respectively. Because of the incomplete sequence of human chromosome 3 genome contig used in this studies, it is recommended that every mutation or polymorphism detected from amplification using this three pairs of primer be reported with respect to HYAL1 gene sequence.

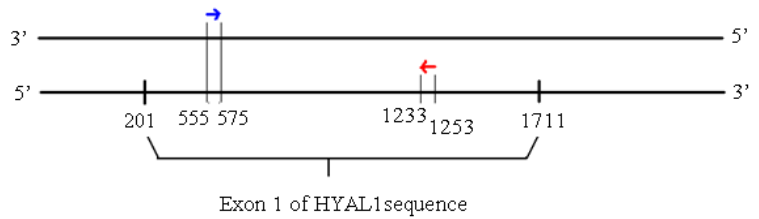

Fig. 13: Annealing region of the first second of primers

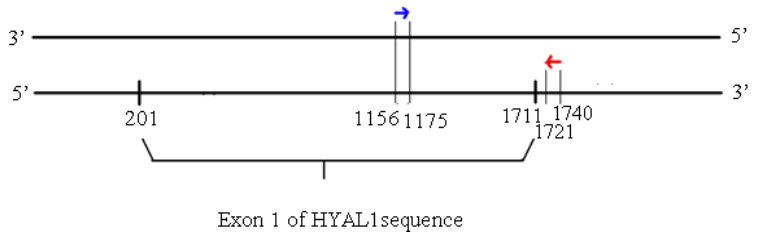

Fig. 14: Annealing region of the third pair of primers

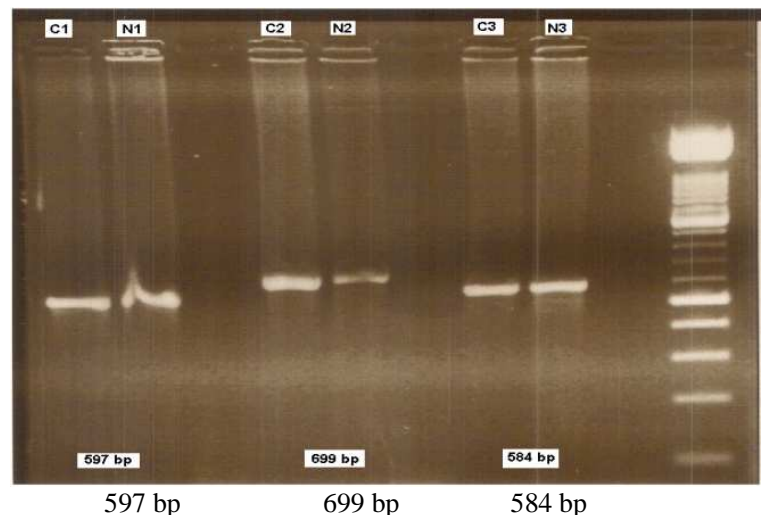

Fig. 15: Photograph image of electrophoresis results conducted to amplified products using the three pair of primers

In order to know whether the three designed pairs of primers were applicable or not to real samples, each pair was put to trial. Three blood samples from healthy individuals were collected and their DNA isolated. Each DNA isolates from these three blood samples were separately amplified by any of these pair of primers. The amplified PCR product using each pair of primers were then subjected to electrophoresis. These resulted in products 597, 699 and 584 bp in size. These is an indication that the three pairs of primers were successfully applicable to real samples as product size of 597, 699 and 584 bp represent product of the first, second and third pairs of primers respectively (Fig. 15).

\section{CONCLUSION}

From the study of exon 1 of HYAL1 gene, the following three pairs of primers may be used: 
For amplifying nt 1-551 of human exon 1 of HYAL1 forward primer 5'TGACCCCCTACAAAAGCTCA-3' (20 bp), rated 86, has 6 homologies to human DNA. Reverse primer 5'AAGTCTCCGATTCCCCCACT-3' (20 bp), rated 92, has 4 homologies with human DNA. For amplifying nt 355-1053 of human exon 1 of HYAL1forward primer 5'-AGTCCTGTGGGAGATGGCAGA-3' (21 bp), rated 91, has 40 homologies to human DNA reverse primer 5'-CGGTAAATGTCCTTGGTGTCC-3' (21 bp), rated 100, has 36 homologies to human DNA. For amplifying nt 956-1511 of human exon 1 of HYAL1 forward primer 5'-GCCATACCTGCTCCTGACTT-3' (20 bp), rated 100, has 30 homologies to human DNA reverse primer 5'-ACAAGGTGGGCAGGTTACAG-3' (20 bp), rated 100, has 34 homologies to human DNA.

When using these three set of primers, nt 1 to 46 of amplified product using the first pair of primers and nt 555-584 of amplified product using the third pair of primers must not be considered because they are not part of exon 1 of HYAL1 gene.

\section{ACKNOWLEDGEMENT}

This research is supported by Ministry of Education (Grant HibahPasca ).This article is dedicated to the memory of Dr. Mardiono Marsetio, Faculty of Medicine, University of Indonesia. The authors are grateful to Dr. Ridla Bakri , Chairman of Department of Chemistry, Faculty of Science, University of Indonesia for his support and critical comments and to Theofilus A. Tockary for his help on preparing the manuscript.

\section{REFERENCES}

Afify, A.M., M. Stern, M. Guntenhoener and R. Stern, 1993. Purification and characterization of human serum hyaluronidase. Arch. Biochem. Biophys., 305: 434-441. PMID: 8373180

Barbara, T.R., T.J. Salo, H. Zhang, B.A. Wicklow and M.R. Natowicz, 1999. Mutations in HYAL1, a member of a tandemly distributed multigene family encoding disparate hyaluronidase activities, cause a newly described lysosomal disorder, mucopolysaccharidosis IX. Proc. Natl. Acad. Sci. USA., 96: 6296-6300. PMID: 10339581

Chao, K., L. Muthukumar and L. Herzberg, 2007. Structure of human hyaluronidase-1, a hyaluronan hydrolyzing enzyme involved in tumor growth and angiogenesis. Biochemistry, 46: 6911-6920. PMID: 17503783
Csoka, T.B., G.I. Frost, H.H.Q. Heng, S.W. Scherer and G. Mohapatra et al., 1998. Thehyaluronidase gene HYAL1 maps to chromosome 3p21.2-p21.3 in human and 9F1-F2 in mouse, a conserved candidate tumor suppresor locus. Genomics, 48: 63-70. PMID: 9503017

Frost, G.I., T.B. Csoka, T. Wong and R. Stern, 1997. Purification, cloning and expression of human plasma hyaluronidase. Biochem. Biophys. Res. Commun., 236: 10-15. PMID: 9223416

Kim, J., M. Johnson, P. Hill and B.K. Gale, 2009. Microfluidic sample preparation: Cell lysis and nucleic acid purification. Integ. Biol., 1: 574-586. DOI : 10.1039/b905844c

Lachaud, L., J. Dereure, E. Chabbert, J. Reynes and J.M. Mauboussin et al., 2000. Optimized PCR using patient blood samples for diagnosis and follow-up visceral leishmaniasis, with special reference to AIDS patients. J. Clin. Microbiol., 38: 236-240. PMID: 10618093

Laurent, T.C., J.R.E. Fraser, U.B.G. Laurent and A. Engstrom-Laurent, 1995. Hyaluronan in inflammatory joint disease. Acta Ortophec., 66: $\quad 116-120$. DOI: $10.1046 /$ j.13652796.1997.00174.x

Liu, D., E. Pearlman, E. Diaconu, K. Guo and H. Mori et al., 1996. Expression of hyaluronidase by tumor cells induces angiogenesis in vivo. Proc. Natl. Acad. Sci. USA., 93: 7832-7837. PMID: 8755562

Shuttleworth, T.L., M.D. Wilson, B.A. Wicklow, J.A. Wilkins and B.L. Triggs-Raine, 2002. Characterization of the murine hyaluronidase gene region reveals complex organization and cotranscription of hyal1 with downstream genes, Fus2 and Hyal3. J. Biol. Chem., 277: 23008-23018. PMID: 11929860

Simpson, M.A. and V.B. Lokeshwar, 2007. Hyaluronan and hyaluronidase in genitourinary tumors. Front Biosci., 13: 5664-5680. PMID: 18508614

Stern, R., 2004. Hyaluronan catabolism: A new metabolic pathway. Eur. J. Cell Biol., 83: 317-325. PMID: 15503855

Wang, F., E.V. Grigorieva, J. Li, V.N. Senchenko and T.V. Pavlova et al., 2008. HYAL1 and HYAL2 Inhibit tumor growth in vivo but not in vitro. Plos One, 3: 1-8. DOI: 10.1371/journal.pone.0003031 